\title{
A LOWER LIMIT TO THE MAGNETIC FIELD IN THE CRAB NEBULA FROM COSMIC $\gamma$-RAY EXPERIMENTS AT $10^{11} \mathrm{eV}$
}

\author{
G. G. FAZIO and H. F. HELMKEN \\ Smithsonian Astrophysical Observatory and Harvard College Observatory, \\ Cambridge, Mass., U.S.A. \\ and \\ G. H. RIEKE and T. C. WEEKES \\ Mt. Hopkins Observatory, Smithsonian Astrophysical Observatory, \\ Amado, Arizona, and Harvard University, Cambridge, Mass., U.S.A.
}

\begin{abstract}
The 10-m optical reflector at Mt. Hopkins, Arizona, has been used to search for cosmic $\gamma$-rays by the detection of atmospheric Cerenkov radiation from energetic particle showers. Approximately 100 drift scans of the Crab Nebula during 1968-69 have yielded no positive evidence of a $\gamma$-ray flux. The upper limit to the flux at $1.7 \times 10^{11} \mathrm{eV}$ is $2.0 \times 10^{-10}$ photons $/ \mathrm{cm}^{2} \mathrm{sec}$. Assuming $\gamma$-rays of this energy are produced by Compton scattering, a lower limit on the average magnetic field in the Crab Nebula is $1.5 \times 10^{-4}$ gauss. This experiment also verifies previous evidence that the high-energy electrons in the Crab Nebula are not the secondary products of high-energy proton interactions but must have been accelerated from lower energies.
\end{abstract}

\section{Introduction}

At energies above $10^{11} \mathrm{eV}$, cosmic $\gamma$-rays can interact with air molecules to generate a cascade of high-energy electrons, positrons, and secondary $\gamma$-rays. This 'air shower' is highly directional and proceeds with relativistic velocity along the direction of the initial $\gamma$-ray photon. The particles in the shower generate a cone of Cerenkov light about $2^{\circ}$ wide along the shower axis. This cone of light will penetrate to sea level with a lateral spread of about $5 \times 10^{4} \mathrm{~m}^{2}$. The duration of the light burst is approximately $10^{-8} \mathrm{sec}$. The detection of this light from $\gamma$-ray showers with energies as low as $10^{11} \mathrm{eV}$ requires a dark site, a cloudless, moonless night, and a large, sensitive light collector. Unfortunately, high-energy cosmic-ray particles also generate air showers and bursts of Cerenkov light in the atmosphere. $\gamma$-rays can be distinguished only by a directional anisotropy in the distribution of Cerenkov light bursts.

\section{Experimental System}

In June 1968, a fully steerable 10-m light reflector was placed in operation at the 2320-m level of Mt. Hopkins, Arizona. The reflector, shown in Figure 1, consists of 248 individually adjustable hexagonal glass mirrors, each with a spherical figure and an aluminized front surface. The focal length of the reflector is $24 \mathrm{ft}(f / 0.7)$, which gives a field of view of about $1^{\circ}$ diameter with a 5 -inch photomultiplier tube at the focus. Additional properties of the detector are given in Table I.

Two operating modes have been used with the $10-\mathrm{m}$ reflector. In one, a single photomultiplier was placed at the focus; the pulses from it were amplified and taken 


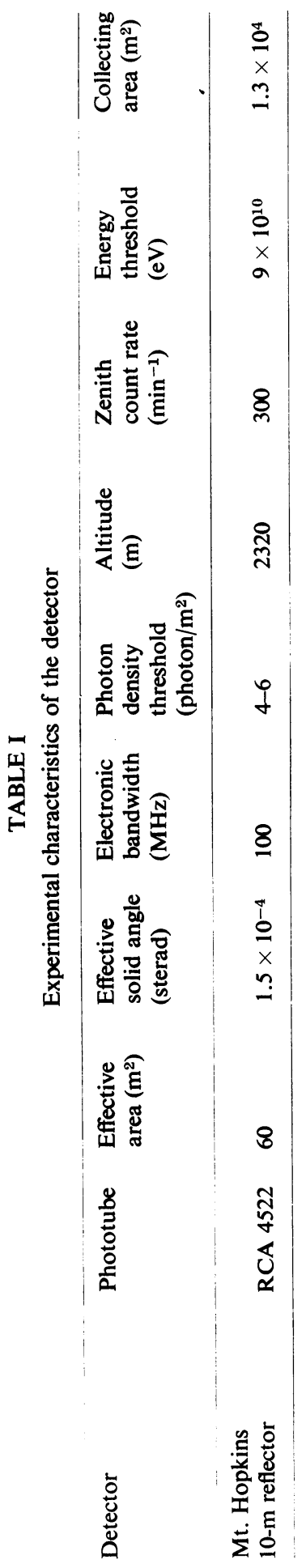




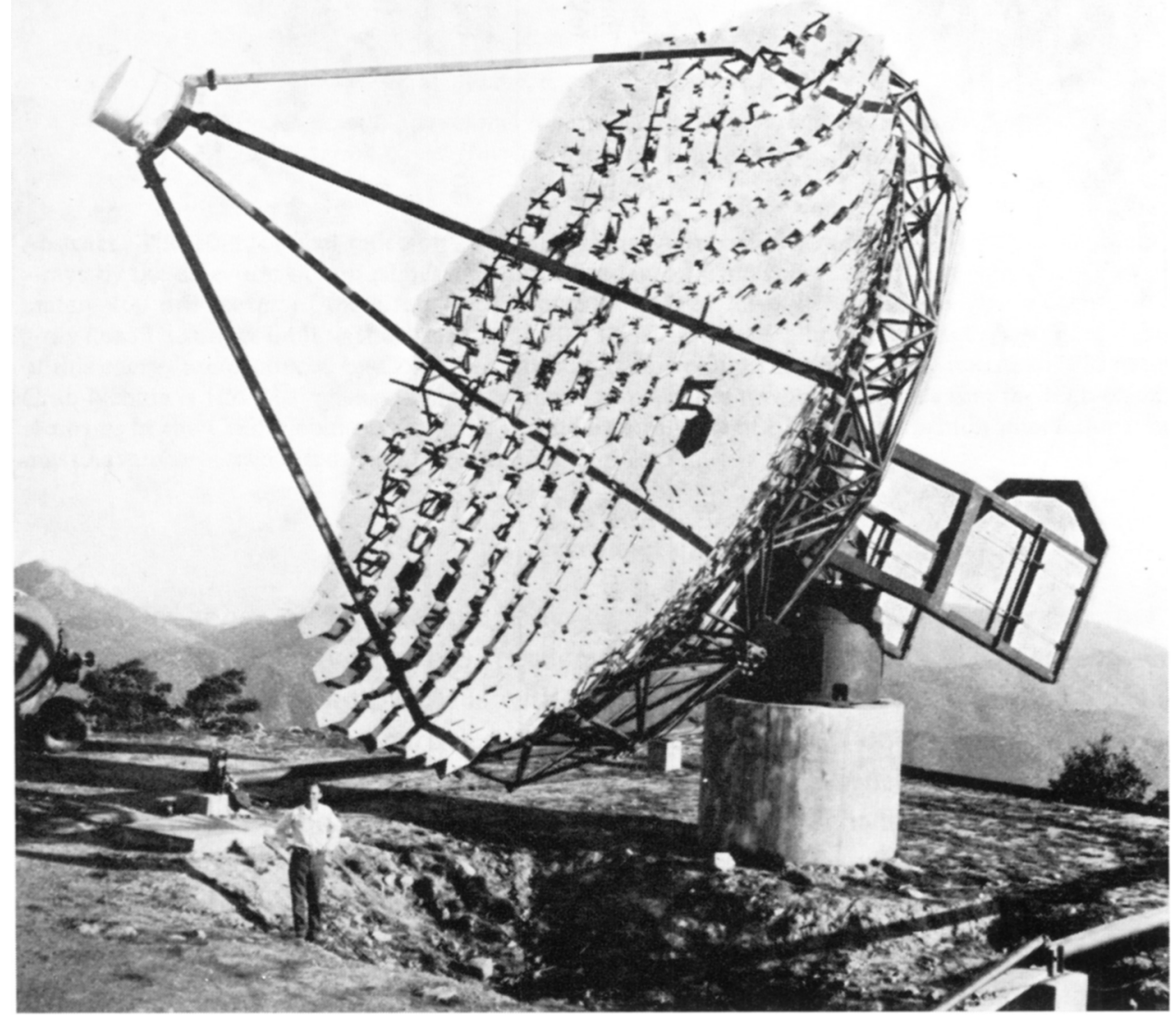

Fig. 1. The 10-m light reflector at Mt. Hopkins, Arizona.

to a discriminator. The discriminator threshold was set high enough so the random contribution to the counting rate was relatively small. The discriminator pulses were counted and printed out automatically. In the second mode, two photomultipliers were mounted on the focus ring of the reflector, with their centers separated by $1 \mathrm{ft}$. For a source on the reflector axis, the outer mirrors were aligned to form an image on one of the photomultipliers, and the inner ones to form an image on the other. The two photomultipliers were then used with a coincidence counting system.

Compared with a single-channel system, a coincidence system has advantages in stability and the ability to work into the noise level of the signal. In the coincidence 
system, the light-pulse broadening introduced by the reflector geometry is reduced from $6 \mathrm{nsec}$ to $3 \mathrm{nsec}$. However, in this case, it has the disadvantage that each photomultiplier sees the whole reflector, including the mirrors aligned for the other phototube. Thus, the background light level is doubled.

A small lamp, driven by a servo-amplifier system, was placed in front of each phototube to keep the photomultiplier output current constant.

The 'drift-scan' technique was used to search for an anisotropy in the shower count rate. This technique consists of positioning the reflector at a point in the sky ahead in right ascension and allowing the earth's rotation to bring the source through the field of view. Depending on the declination, the duration of each scan is about $40 \mathrm{~min}$, centered on the transit of the suspected source.

\section{Observations}

More than 14 possible sources of cosmic $\gamma$-rays have been investigated to date with the $10-\mathrm{m}$ reflector. However, this paper will be concerned only with the data collected on the Crab Nebula. During the moonless periods from September 1968 to December 1969, 102 drifts scans were made on the Crab Nebula under excellent sky conditions.

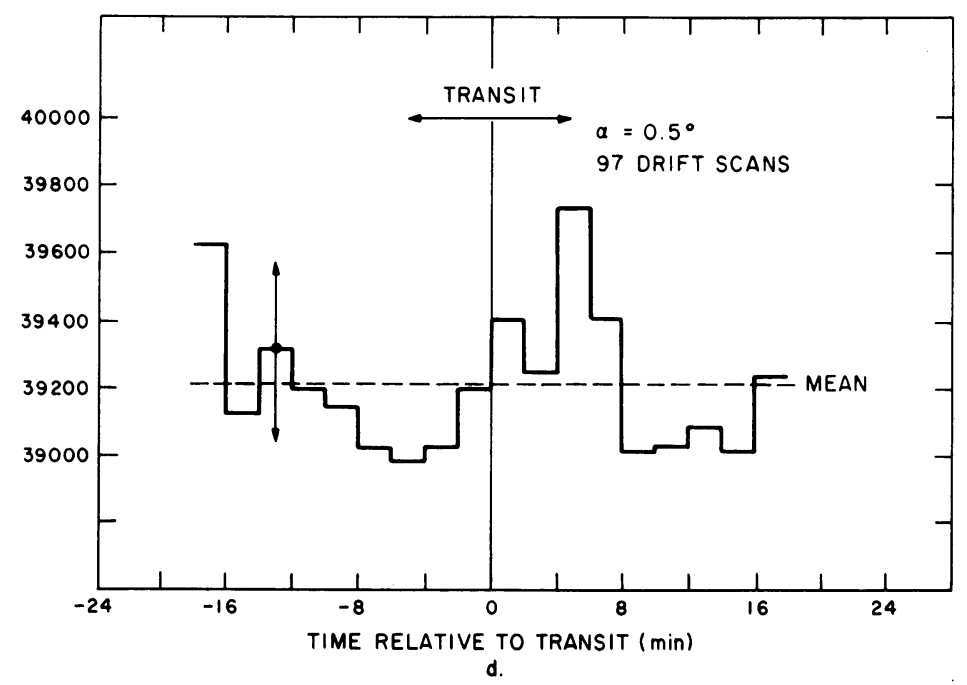

Fig. 2. Histogram showing the cumulative counts from drift scans on the Crab Nebula.

A histogram summarizing the results of these drift scans is shown in Figure 2. In order to have a consistent rule for the rejection of scans in which nonrandom shifts in the counting rate occurred, any drift scan is rejected in which there is a statistical probability of less than $1 \%$ that the counting rate observed before the source entered the field of view is the same as the rate after it left the field. Five drift scans were 
rejected due to this criterion. A crude analysis of the accepted scans indicates that the observed fluctuations are $15 \%$ greater than those expected from statistical arguments. Therefore, the error quoted for the data is 1.15 times the standard deviation, and the flux limit is based on this error.

\section{Results}

As shown in Figure 2, there is no apparent anisotropy in the shower count rate greater than that expected from detection of a random angular distribution of showers. The upper limit (three standard deviations) to the cosmic $\gamma$-ray flux at energies greater than $1.7 \times 10^{11} \mathrm{eV}$ from the Crab Nebula is $2 \times 10^{-10}$ photons $/ \mathrm{cm}^{2} \mathrm{sec}$. The observations were taken at an average zenith angle of $25^{\circ}$, and the excess counting rate from the source, subtracting background, was $+0.16 \pm 0.68 \mathrm{~min}^{-1}$. The source was in the field of view for a total of $776 \mathrm{~min}$. In Figure 3, this upper limit is plotted along with the observations of other groups. The $\gamma$-ray threshold energy for this experiment is an order of magnitude lower than that of previous experiments.

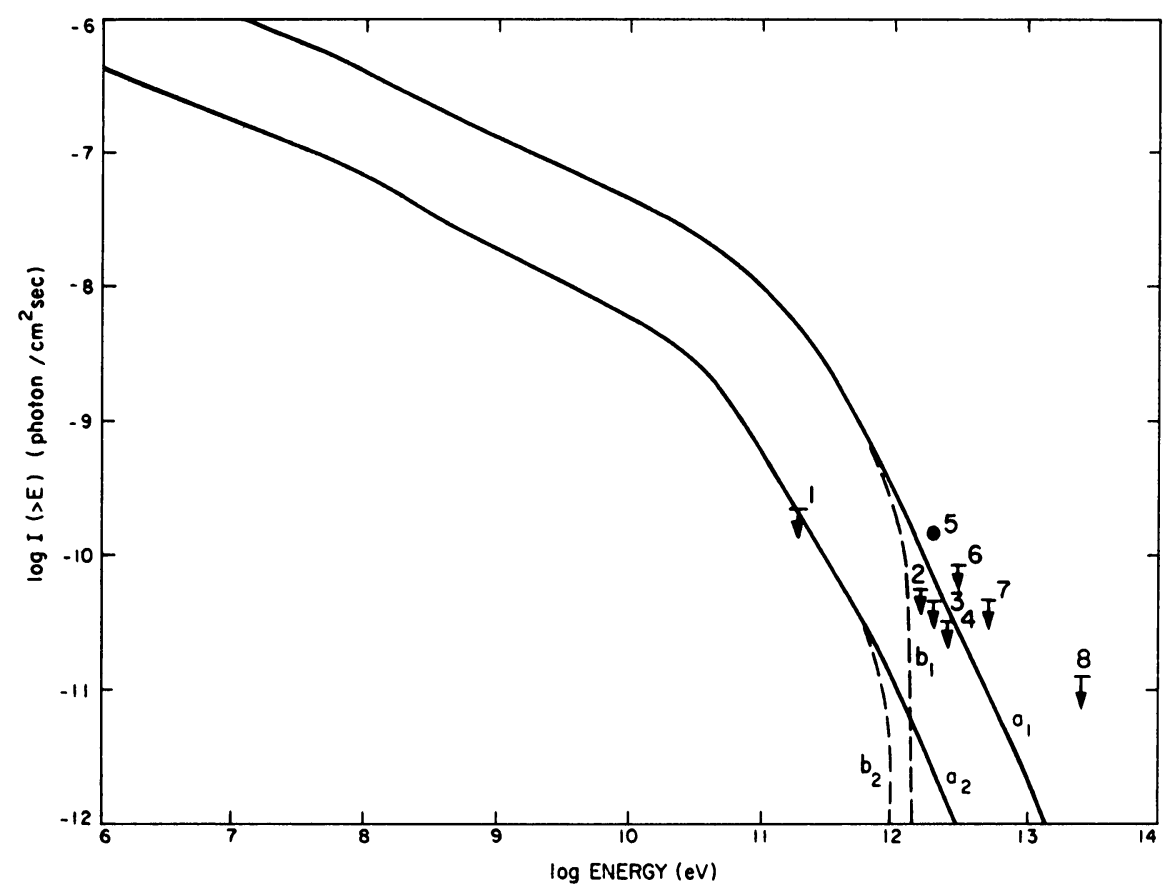

Fig. 3. Inverse Compton $\gamma$-ray flux from the Crab Nebula. Curves $a_{1}$ and $b_{1}: H=10^{-4}$ gauss. Curves $a_{2}$ and $b_{2}: H=3 \times 10^{-4}$ gauss. Curves $a_{1}$ and $a_{2}$ were computed assuming that the X-ray flux is synchrotron radiation; for $b_{1}$ and $b_{2}$, it was assumed that the synchrotron spectrum cuts off at $10^{16} \mathrm{~Hz}$. Experimental point: 5, Fegan et al. (1968). Experimental upper limits: 1, this paper; 2-4, Rieke (1969), Fazio et al. (1968); 6, Tornabene and Cusimano (1968); 7, Chudakov et al. (1965); 8, Long et al. (1965). 


\section{Interpretation of the Results}

Gould (1965) and Rieke and Weekes (1969) have calculated the $\gamma$-ray energy spectrum from the Crab Nebula, taking into account Compton scattering in the source between the synchrotron electrons and the observed synchrotron-emitted photons. The $\gamma$-ray flux predicted is a sensitive function of the magnetic field:

$$
F\left(E_{\gamma}\right) \propto H^{-(1+m) / 2},
$$

where $m$ is the spectral index of the relevant electron power-law spectrum. For the Crab Nebula, in the energy range of interest, the value of $m$ is about 3.2; hence,

$$
F\left(E_{\gamma}\right) \propto 1 / H^{2 \cdot 1} \text {. }
$$

Apparao (1967) has extended these calculations to include the collision of synchrotron electrons within the Crab Nebula with the photons of the $3 \mathrm{~K}$ universal blackbody radiation. These three calculations are the most accurate predictions of a $\gamma$-ray flux from the Crab Nebula that exist. Figure 3 shows the results of Rieke and Weekes (1969) for two values of the magnetic field. From this figure, it appears that the upper limit set by this experiment is sufficient to place a lower limit of $3 \times 10^{-4}$ gauss on the strength of the magnetic field in the Crab Nebula. However, in view of the uncertainties in the experimental flux, the energy-threshold estimates, and the approximations in the theoretical calculations, a more reasonable lower limit is about $1.5 \times 10^{-4}$ gauss. It should be emphasized that this limit does not depend on the extrapolation of the optical synchrotron spectrum to the X-ray region. A commonly accepted value for the field strength is $10^{-4}$ gauss.

One of the principal results from less sensitive experiments is evidence that the highenergy electrons in the Crab Nebula are not the product of high-energy proton interactions and therefore must have been accelerated from lower energies (Cocconi, 1960; Gould and Burbidge, 1967; Chudakov et al., 1965). The greater sensitivity of the 10-m reflector allows this result to be verified even more conclusively.

This experiment does not confirm possible evidence for a $\gamma$-ray flux from the Crab Nebula at $2 \times 10^{12} \mathrm{eV}$ reported by Fegan et al. (1968).

Several groups are attempting to detect $\gamma$-rays with an energy of $10^{8} \mathrm{eV}$ from the Crab Nebula. The most realistic predictions of this flux of photons are also given by the Compton-scattering calculations of Gould (1965), Apparao (1967), and Rieke and Weekes (1969). Assuming a magnetic-field intensity of $3 \times 10^{-4}$ gauss in the Crab Nebula, the predicted flux is now about $10^{-7}$ photons $/ \mathrm{cm}^{2} \mathrm{sec}$, an order of magnitude lower than earlier predictions.

The pulsating radio source (NP0532) in the center of the Crab Nebula was also investigated for high-energy $\gamma$-ray emission, and the results are reported in another paper in this symposium.

\section{Acknowledgements}

We wish also to acknowledge the assistance of Roger Carson, Don Hogan, and Ed Horine during this experiment. 


\section{References}

Apparao, M. V. K.: 1967, Proc. Indian Acad. Sci. 65A, 349.

Chudakov, A. E., Dadykin, V. L., Zatsepin, V. I., and Nesterova, N. M.: 1965, in Proc. (Trudy), Lebedev Physics Inst. (ed. by D. V. Skobel'tsyn), Consultants Bureau, New York, 26, 99.

Cocconi, G.: 1960, Proc. Intl. Conf. Cosmic Rays, Moscow, 2, 309.

Fazio, G. G., Helmken, H. F., Rieke, G. H., and Weekes, T. C.: 1968, Astrophys. J. (Letters) 154, L83.

Fegan, D. J., McBreen, B., O'Mongain, E. P., Porter, N. A., and Slevin, P. J.: 1968, Canadian J. Phys. 46, S433.

Gould, R. J.: 1965, Phys. Rev. Letters 15, 577.

Gould, R. J. and Burbidge, G. R.: 1967, Handbuch der Physik 46/II, 265.

Long, C. D., McBreen, B., Porter, N. A., and Weekes, T. C.: 1965, Proc. Intl. Conf. Cosmic Rays, London 1, 318.

Rieke, G. H.: 1969, Smithson. Astrophys. Obs. Spec. Rep. No. 301.

Rieke, G. H. and Weekes, T. C.: 1969, Astrophys. J. 155, 429.

Tornabene, H. S. and Cusimano, F. J.: 1968, Canadian J. Phys. 46, S81. 